\title{
Thoracophelia flabellifera n. sp., ein neuer sedentärer Polychät (Familie Opheliidae) in der Deutschen Bucht
}

\author{
Von Erich Ziegelmeier \\ Aus der Biologischen Anstalt Helgoland, List auf Sylt, \\ in der Bundesforschungsanstalt für Fischerei \\ (Mit 2 Abbildungen und 2 Tabellen)
}

Nachdem bereits im Frühjahr und Herbst 1952 bei quantitativen Bodenfaunauntersuchungen das Vorkommen der beiden für die Deutsche Bucht neuen Polychäten Capitomastus minimus (LANGERHANs) und Goniada bobrezkii (ANNEnkova) festgestellt wurde (Ziegelmeier, 1953), konnte in den Fängen vom Frühjahr 1953 und Herbst 1954 eine noch nicht beschriebene Art aus der Familie der Opheliidae nachgewiesen werden.

Die kleinen Polychäten fallen besonders auf durch die Zonierung des Körpers in einen Kopfabschnitt, einen thorakalen und einen abdominalen, kiementragenden Teil. Danach sind die hier vorliegenden Tiere zur Gattung Thoracophelia zu rechnen, die von EHLERs (1897) mit der Art Th. furcifera nach einigen Exemplaren, die im "Sande des tiefsten Ebbestrandes" in der Magalhaes-Straße (b. Punta Arenas) gesammelt wurden, aufgestellt worden ist.

Die 3-Teilung des Wurmkörpers ist das wichtigste Merkmal, das die Gattung Thoracophelia (EHLERs) vom Genus Ophelia (SavignY) unterscheidet: Durch eine ringförmige Einschnürung ist ein Kopfabschnitt deutlich vom thorakalen Teil abgesetzt

$\mathrm{Da}$ die hier gefundenen Polychäten auch in anderen diagnostischen Merkmalen, vor allem in der gegabelten Form der Kiemen, mit Thoracophelia ungefähr übereinstimmen, ist die Einreihung der vorliegenden Opheliide in diese Gattung berechtigt. Gegabelte Kiemen sind außer bei der Gattung Thoracophelia nur für Ophelia asworthi (FAUveL, 1917) beschrieben worden.

Bisher sind 45 Exemplare von Thoracophelia flabellifera $n$. sp., jedoch nur an einer Stelle der Deutschen Bucht - Station: "Tonne 7" (frühere Bezeichnung "Tonne $\left.3 \mathrm{~A}^{\prime \prime}\right)$, querab Hörnum/Sylt, Position: $54^{0} 42,5^{\circ} \mathrm{N}-8^{0} 5,0^{\circ} \mathrm{O}$, $13 \mathrm{~m}$ Tiefe - gefunden worden. 10 Tiere konnten lebend beobachtet werden (Abb. 2 A-C).

Bemerkenswert ist die Substratgebundenheit von Th. flabellifera $n$. sp. und ihr stets gemeinsames Vorkommen mit Travisia forbesii in braungelbem, reinem, etwas grobkörnigerem Sand. Ihre ungefähre Abundanz ergibt folgende Zusammenstellung: 


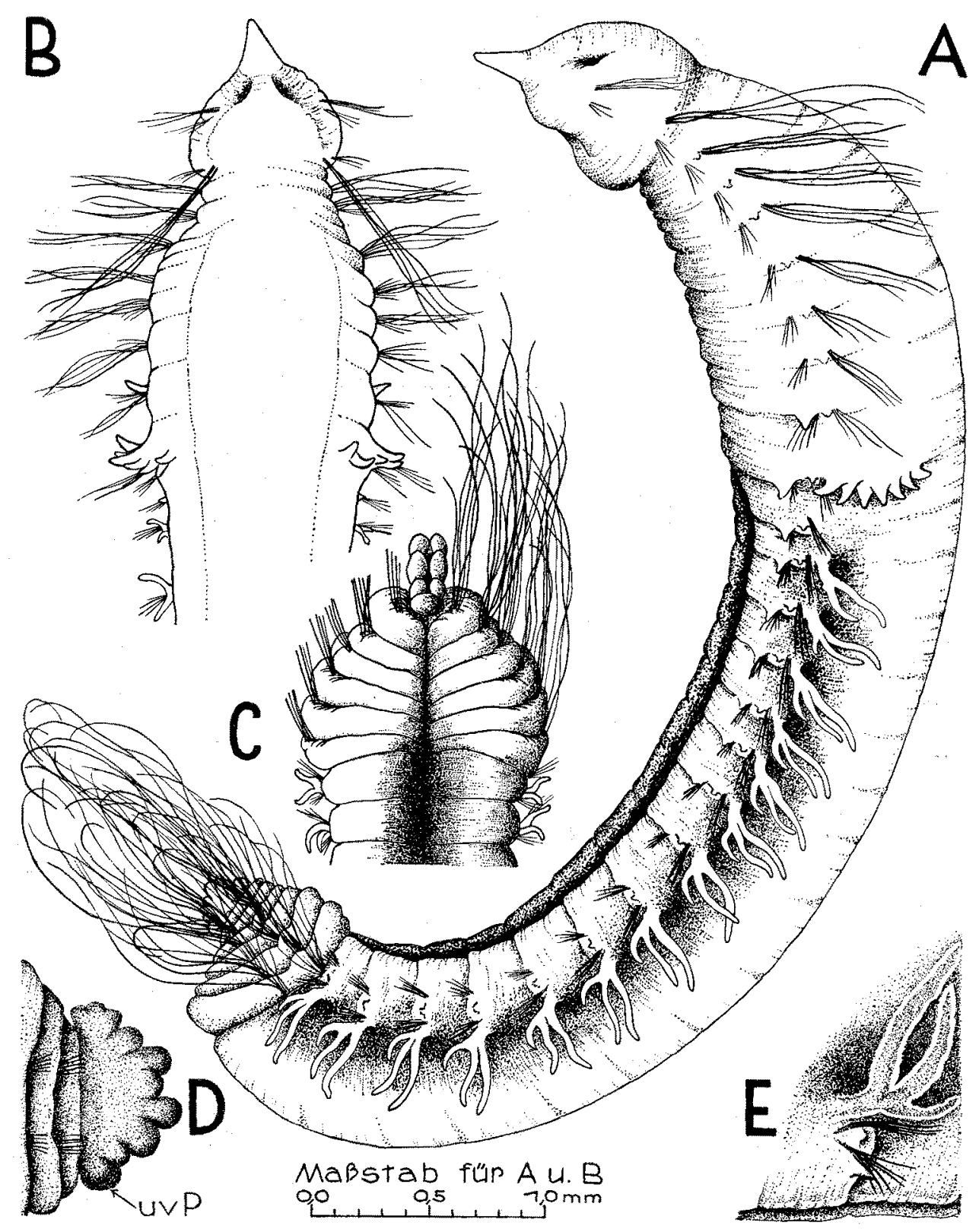

Abb. 1. $\mathrm{A}=$ Thoracophelia flabellifera $n . s p$. Totalansicht von der linken Körperseite. $B=$ Kopfabschnitt und thorakaler Teil, dorsale Ansicht. $\mathrm{C}=$ Hinterende, ventral. Die letzten kiementragenden und die 6 präanalen Segmente mit dem Pygidium. Die rechten präanalen Borstenbüschel sind nur angedeutet und nicht in ihrer gesamten Länge eingezeichnet worden. $\mathrm{D}=$ Analsegment von der linken Seite (uvP = unpaare, ventrale Papille) mit den beiden letzten präanalen Segmenten, bei denen die noto- und neuropodialen Borstenbündel ebenfalls nur am Ansatz angedeutet gezeichnet wurden. $\mathrm{E}=$ linke Kieme aus der vorderen Region des abdominalen Teils mit Ventralcirrus und dem kegelstumpfförmigen, kleinen Organ zwischen dem ventralen und dorsalen Borstenbüschel. 
Tabelle 1

$\begin{array}{cc}\text { Datum der Probeentnahme } & \text { Durchschnittliche Anzahl auf } 0,1 \mathrm{qm} \text { b. Tonne } 7 \\ \text { 14. } 5.53 & 3 \\ 1.9 .54 & 15 \\ 20.11 .54 & 10\end{array}$

Die Farbe der lebenden Tiere ist rosa-fleischfarben, etwa wie bei Ophelia limacina, die der mit Formalin fixierten blaßgelb bis farblos.

Die Länge der untersuchten Würmer beträgt 3,8 bis $9,0 \mathrm{~mm}$, ihre Dicke 0,6 bis $1,2 \mathrm{~mm}$. Ob es sich bei den bisher gefundenen um junge Tiere handelt, war nicht eindeutig zu klären, da bei keinem der Würmer Geschlechtsprodukte beobachtet werden konnten. Die Vermutung liegt jedoch nahe, daß die längsten der vorliegenden Polychäten ausgewachsen sind, weil die Größen der im Frühjahr und Herbst gesammelten Tiere keine Unterschiede aufwiesen.

Nach Kükenthal (1889) muß „bei den Opheliiden die Konstanz der Segmente in derselben Art als charakteristisches Merkmal angesehen werden" Bei der vorliegenden neuen Art sind stets 32 Segmente zu zählen, die sich auf die einzelnen Körperregionen wie folgt verteilen: Kopfabschnitt $=2 \mathrm{Seg}$ mente, Thorax $=8$, abdominaler Teil $=21$ und 1 Analsegment (Abb. $1 \mathrm{~A}$ ).

Die Ringelung der einzelnen Segmente ist am Vorderende noch gut $\mathrm{zu}$ erkennen (Abb. 2 C), wird aber hinter dem Kopfabschnitt, im thorakalen und abdominalen Teil undeutlich. Schwach granulierte Streifen, die vor allem im mittleren Körperabschnitt vom Rücken des Wurmes nach beiden Seiten hin spitz zulaufen, deuten dorsal die Segmentgrenzen an. Ventral sind im abdominalen Teil die Segmente durch Querfurchen gegeneinander deutlich abgegrenzt.

Das Prostomium hat die für die meisten Opheliiden typische, konisch-zugespitzte Form. Der Rüssel ist in ausgestülptem Zustand blasig aufgetrieben (Abb. $2 \mathrm{~A})$.

Der Kopfabschnitt besteht aus 2. Segmenten, von denen das erste das Prostomium, ventral die Mundöffnung und zu beiden Seiten je eine Nuchalgrube besitzt. Etwa in der Höhe der Mundöffnung stehen lateral die ersten beiden Borstenbüschel. An der ringförmigen Einschnürung, der Grenze zwischen dem Kopfabschnitt und dem thorakalen Teil, befindet sich das 2. Borstenbüschelpaar mit langen Borsten im Notopodium. Sämtliche Parapodien sind zweiästig, die dorsalen Borsten sind stets (mehr oder weniger erkennbar) länger als die des ventralen Astes.

Der sich an den Kopfabschnitt anschließeade thorakale Teil besteht aus 8 Segmenten (3. bis 10. Körpersegment). Die Borstenbündel sind gegenüber denen des Kopfteiles ein wenig ventralwärts gerückt. Bis zum letzten thorakalen Segment werden die notopodialen Borsten kontinuierlich kürzer. Vom ersten Thoraxsegment (3. Körpersegment) an ist zwischen den beiden Parapodästen ein sehr kleiner kegelstumpfförmiger Höcker zu erkennen, der sich bis zum letzten kiementragenden Segment verfolgen läßt und wohl mit dem von FAUveL (1917) als "pore sensitif ovale" und von OKUDA (1934) als "slit like sensory pits" bezeichneten Organ identisch ist. Am vorletzten und letzten Thoraxsegment (9. und 10. Körpersegment) sowie am ersten abdominalen Segment (11. Körpersegment) ist im Neuropodium ein Ventralcirrus ausgebildet. Während die drei erwähnten Segmente den Ventralcirrus stets besitzen, ist er bei einigen Würmern (meist bei größeren Tieren) bis zum 4 . oder 5 . kie- 
mentragenden Segment festzustellen, wird jedoch immer kürzer und verschwindet schließlich ganz.

Auffallend sind die zu beiden Seiten am letzten Thoraxsegment (10. Körpersegment) über dem dorsalen Borstenbündel stehenden vertikalen Reihen von 5 bis 7 kurzen und kegelförmigen Cirren, die, fächerartig oder etwa gespreizten Fingern gleich, die Grenze zwischen dem thorakalen und abdominalen Körperabschnitt markieren. Hierin weicht nun Th. flabellifera $n . s p$. von den bisher beschriebenen Arten der Gattung ab, bei denen am letzten Thoraxsegment an dieser Stelle je ein vertikaler Wulst (nach O. Hartman, 1938 "glandular ridge" und nach ОкuDA, 1934 "lateral ridge") zu erkennen ist. Am 10. Körpersegment beginnt auch die für Ophelia typische Ventralfurche. Vom letzten thorakalen Segment ab sind Ventralcirrus und Borstenbündel ein wenig ventralwärts verschoben.

Während Kopf- und thorakaler Teil drehrund sind, erfährt im abdominalen Körperabschnitt die zylindrische Form durch die Ventralfurche und die Furchen, die zu beiden Seiten über den Borstenbüscheln, in der Höhe der Ansatzstellen der Kiemen entlanglaufen, eine Abänderung. Der abdominale Teil des Wurmkörpers besteht aus zwei deutlich voneinander zu unterscheidenden Abschnitten, 1. den kiementragenden und 2. den postbranchialen oder präanalen Segmenten mit dem Pygidium. Das erste Segment des Abdomens ist kiemenlos, die Kiemen beginnen am darauffolgenden, also stets am zweiten Abdominalsegment (12. Körpersegment). Ihre Anzahl ist konstant, es konnten bei allen untersuchten Tieren immer 14 Kiemenpaare gezählt werden.

Die Kiemen, die unmittelbar über den notopodialen Borsten ansetzen, sind gegabelt. Die Anzahl der Gabeläste (höchstens 3) ändert sich einmal beim gleichen Tier, indem das vorderste Paar stets einen Ast weniger besitzt als die folgenden. Ab 2. Kiemenpaar ist ein weiterer Ast, nach dem Hinterende zu immer größer werdend, zu erkennen. Weiterhin ist die Ausbildung der Gabelform sehr wahrscheinlich mit dem Wachstum verbunden, denn die Astzahl wird mit zunehmender Länge der Tiere größer. Bei sehr kleinen Würmern konnten auch einfache, nicht gegabelte Kiemen beobachtet werden (vergl. Tab. 2).

Während die Borsten im abdominalen Teil kurz sind, tragen die dem Kiemen-Abschnitt folgenden 6 präanalen Segmente sehr lange Kapillarborsten, die dichte Haarfächer um das Analsegment bilden. Gegenüber den vorangegangenen Borsten der Kiemensegmente sind die Büschel des Wurmhinterendes etwas dorsalwärts verlagert. Die beiden vordersten der 6 postbranchialen Segmente sind nur wenig länger als die 4 folgenden, die, etwas ineinandergeschoben, dorsal eine rinnenartige Einbuchtung erkennen lassen. Die abdominale, tiefe Ventralfurche setzt sich auf der Ventralseite der präanalen Segmente in einer schmalen, konvergierenden Rinne bis zum Pygidium fort (Abb. 1 C).

Das Analsegment besteht aus zwei, den dorsoventralstehenden Afterspalt flankierenden Fächern, deren Außenrand von 7 kleinen, in ihrer kugeligen Form etwas variierenden Papillen gebildet wird. Ventral befindet sich eine kleine unpaare, abgerundete Papille (Abb. $1 \mathrm{C}$ und D).

Bei den lebenden Würmern ( $\mathrm{Abb}$. $2 \mathrm{~A}$ und $\mathrm{C}$ ) fallen besonders die abgeflachten, runden Zellen im Körperinneren auf, die bei den Bewegungen der Tiere mit der strömenden Körperflüssigkeit ständig transportiert werden. 

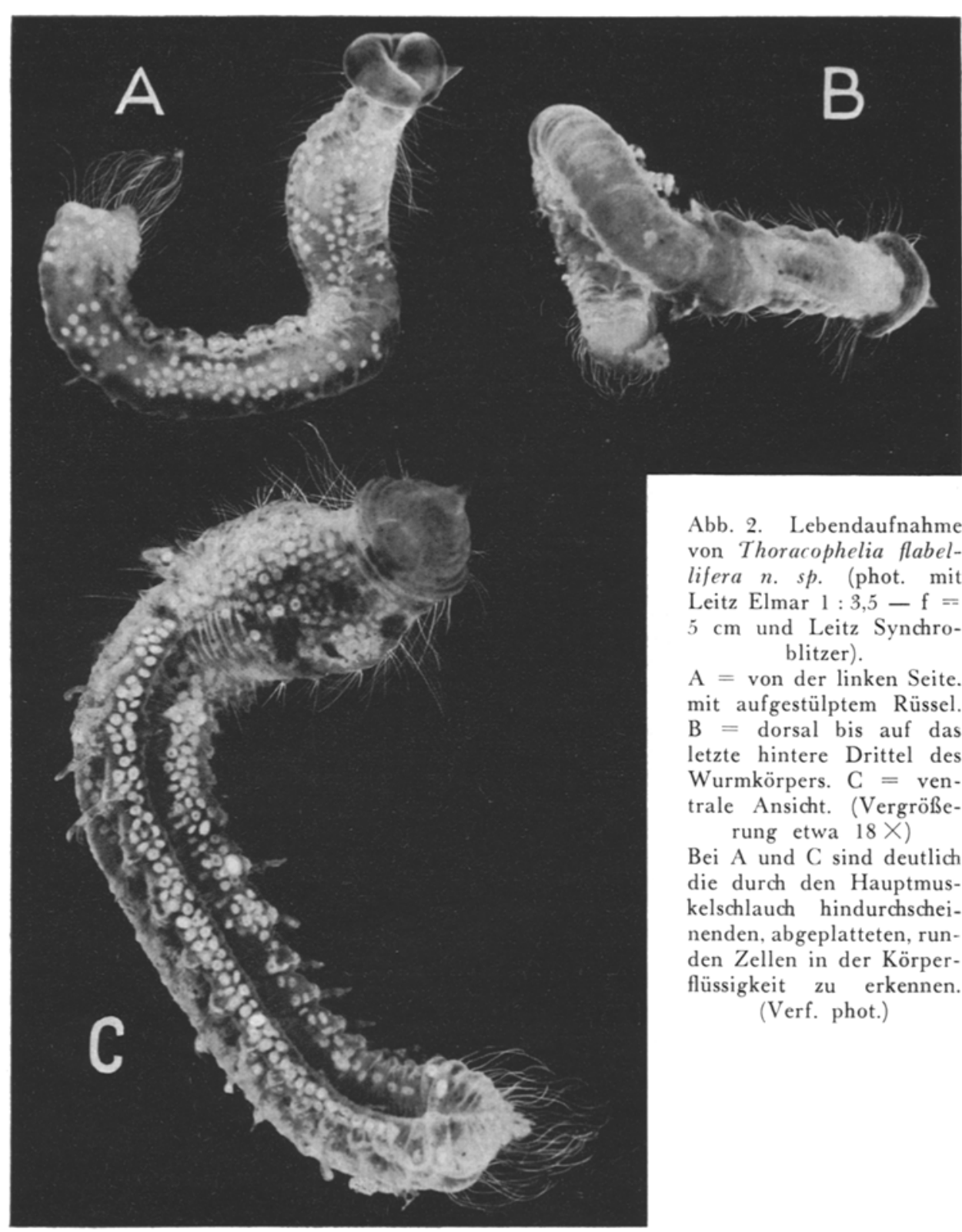

Abb. 2. Lebendaufnahme von Thoracophelia flabellifera n. sp. (phot. mit Leitz Elmar 1 : 3,5 - $\mathrm{f}=$ $5 \mathrm{~cm}$ und Leitz Synchroblitzer).

$A=$ von der linken Seite. mit aufgestülptem Rüssel. $\mathrm{B}=$ dorsal bis auf das letzte hintere Drittel des Wurmkörpers. $\mathrm{C}=$ ventrale Ansicht. (Vergrößerung etwa $18 \times$ )

Bei $\mathrm{A}$ und $\mathrm{C}$ sind deutlich die durch den Hauptmuskelschlauch hindurchscheinenden, abgeplatteten, runden Zellen in der Körperflüssigkeit zu erkennen. (Verf. phot.)

Die konstanten Merkmale bei den bisher gefundenen Exemplaren von Thoracophelia flabellifera n. sp. sind:

1. Gesamtzahl der Segmente $=32$

2. Gleichbleibende Segmentzahlen in den einzelnen Körperabschnitten

3. 14 Kiemenpaare

4. Beginn der Kiemen am 12. Körpersegment

5. Mindestzahl der Ventralcirren $=3$, die sich am 9., 10. und 11. Körpersegment befinden

6. Anzahl der lateralen Papillen im Pygidium $=7$ Paare. 
Die variablen Merkmale sind in der folgenden Tabelle auf Grund der Untersuchung von 22 vollkommenen und unbeschädigten Tieren zusammengestellt worden:

Tabelle 2

\begin{tabular}{llllllllllllllllllllllllll}
\hline Länge (in mm) & 3,8 & 4,3 & 4,6 & 4,9 & 5,0 & 5,0 & 5,1 & 5,2 & 5,4 & 5,9 & 6,0 & 6,0 & 6,1 & 6,5 & 7,0 & 7,0 & 7,0 & 7,7 & 7,8 & 7,8 & 8,0 & 9,0 \\
\hline $\begin{array}{l}\text { Dicke } \\
\text { (in morax }\end{array}$ & 0,8 & 0,8 & 0,9 & 1,0 & 0,9 & 0,9 & 0,9 & 0,8 & 0,8 & 1,0 & 0,9 & 0,9 & 1,0 & 1,0 & 1,0 & 1,1 & 1,0 & 1,2 & 1,2 & 1,2 & 1,2 & 1,0 \\
\hline $\begin{array}{l}\text { Anzahl der } \\
\text { Gabeläste } \\
\text { der Kiemen }\end{array}$ & 1 & 2 & 1 & 2 & 2 & 2 & 2 & 2 & 2 & 2 & 2 & 2 & 2 & 3 & 2 & 2 & 2 & 3 & 3 & 3 & 3 & 3 \\
\hline $\begin{array}{l}\text { Anzahl d. dors. } \\
\begin{array}{l}\text { Cirren am } \\
\text { lo. Körpersegment }\end{array}\end{array}$ & 6 & 5 & 6 & 5 & 7 & 5 & 7 & 6 & 6 & 6 & 6 & 6 & $\begin{array}{l}6 \\
5\end{array}$ & li. & 6 & 7 & 6 & 6 & 7 & 7 & 7 & 7 & 7 \\
\hline $\begin{array}{l}\text { Anzahl der } \\
\text { Ventralcirren }\end{array}$ & 3 & 6 & 4 & 5 & 4 & 5 & 5 & 6 & 4 & 6 & 3 & 7 & 6 & 7 & 6 & 7 & 7 & 7 & 7 & 5 & 6 & 8 \\
\hline
\end{tabular}

Merkmale, in denen die hier festgestellte neue Art von den Arten der Gattung Thoracophelia (EHLERs) a bw eichen, sind:

1. Besitz der dorsalen Cirrenfächer am 10. Körpersegment anstelle der lateralen Wülste

2. Vorhandensein von mindestens 3 Ventralcirren

3. Beginn der Kiemenpaare am 12. Körpersegment (bei den beiden anderen Arten der Gattung am 13. Segment)

4. Bau des Pygidiums.

Die Frage, ob die kleinen Polychäten bisher übersehen wurden oder ob es sich bei der Opheliide um eine der Arten handelt, die infolge der Erwärmung der nordatlantischen Géwässer in unseren Meeresteil eingewandert sind, ist sehr schwer zu beantworten. Vielleicht könnte die Beobachtung, nach der die mit Travisia forbesii im ungeheizten Raum zusammen gehälterten Tiere bei den tiefen Temperaturen im Januar 1955 zugrunde gingen, während Travisia am Leben blieb, darauf hindeuten, daß Thoracophelia flabellifera n. $s p$. eine wärmeliebendere Art ist.

\section{Diagnose}

(Holotypus in der Sammlung der Biologischen Anstalt Helgoland in List auf Sylt - Nr. 55/1)

Gesamtzahl der Segmente $=32-$ Drei Körperregionen: Kopfabschnitt $=2$ Segm., Thorax $=8$ Segm., abdominaler Teil $=21$ Segm. - Pygidium Prostomium konisch zugespitzt - Erstes Körpersegment, das Peristomium mit Mundöffnung, 2 Nukalorganen und den ersten beiden Borstenbüscheln - Am 10. Körpersegment, an der Grenze zwischen Thorax und Abdomen, zu beiden Seiten vom notopodialen Borstenbüschel dorsalwärts je ein Fächer von 7 kurzen, kegelförmigen Cirren - Ventralfurche ab 10. Körpersegment - Je ein kleines kegelstumpfförmiges Organ zwischen den Parapodästen vom 3. bis 25. Körpersegment - 14 Kiemenpaare - Kiemen gegabelt mit 2 u. 3 Gabelästen -6 postbranchiale oder präanale Segmente mit langen Borsten $-\mathrm{Py}$ - 
gidium mit 7 Paar kleinen, kugeligen, lateralen Papillen, eine ventrale, unpaare Papille.

Länge $=8 \mathrm{~mm}$, Dicke $=1,2 \mathrm{~mm}$.

Die Gattung Thoracophelia war nach der Aufstellung durch EHLERs (1897) nur mit einer Art, Th. furcifera (vgl. S. 251) vertreten, die als Genotypus nach einigen Exemplaren eines Fundortes beschrieben wurde. Weitere, die Existenz der Gattung sichernde Fundortangaben fehlten. Erst 1938 konnte in die Gattung Thoracophelia von O. Hartman eine weitere Art, Th. mucronata, eingereiht werden, die TrEadwell (1914) als Ophelina mucronata beschrieb, die jedoch wegen der 3-Teilung des Wurmkörpers und der Gabelform der Kiemen zur Gattung Thoracophelia (EhLERs) gehört. Als 3. Art tritt nun Th. flabellifera $n$. sp. hinzu, die sich wie folgt in den von O. Hartman (1938) angegebenen Bestimmungsschlüssel der Arten für die Gattung Thoracophelia (EHLERs) einbeziehen läßt:

20 Paar Kiemen und 6 postbranchiale Segmente; Pygidium mit 5 Paar dorsalen Papillen . . . . . . . Th. furcifera (EhLERs)

18 Paar Kiemen und 8 postbranchiale Segmente; Pygidium mit 7 Paar dorsalen Papillen . . . . . . Th. mucronata (TREADWELL)

14 Paar Kiemen und 6 postbranchiale Segmente; Pygidium mit 7 Paar kleinen, lateralen Papillen . . . Th. flabellifera n. sp.

\section{Literatur}

Ehlers, E., 1897: Polychaeten. Hamburger Magalhaensische Sammelreise (Hamburg, Friedrich \& Co.).

Fauve1, P., 1917: Annelides polychètes de l'Australie méridionale. Arch. de Zool. exp. et gén. 56.

F a uve 1, P., 1927: Polychètes sedentaires. Faune de France, 16, Paris.

Friedrich, H., 1938: Polychaeta. Tierw. Nord- u. Ostsee, Teil VI b.

Hartman, Olga, 1938: Descriptions of new species and new generic records of polydaetous annelids from California of the families Glyceridae, Eunicidae, Stauronereidae and Opheliidae. Univ. Calif. Publ. Zool. 43, 6.

Kükenthal, W., 1889: Beiträge zur Fauna Spitzbergens, Resultate einer im Jahre 1886 unternommenen Reise. I. Teil. Archiv für Naturgeschichte, 55 (1. Band).

Okuda, S., 1934: Description of a new polychaete Thoracophelia yasudai. Journ. Fac. Sci. Hokkaido Imperial Univ., ser. 6, 3.

Treadwell, A. L., 1914: Polychaetous annelids of the Pacific Coast in the collection of the zoological museum of the University of California. Univ. Calif. Publ. Zool., 13.

Ziegelmeier, E., 1953: Kurze Mitteilung über zwei für die Deutsche Bucht neue Polychätenarten der Gattungen Capitomastus und Goniada. Helg. Wiss. Meeresunt. 4. 